\title{
Evaluation of the Practice of Antibiotic Prophylaxis in Trauma and Orthopedic Surgery at the Moulay Ismail Military Hospital in Meknes
}

\author{
El Kartouti Abdeslam ${ }^{1 *}$, Sidi Mohamed Hannafi ${ }^{2}$, My Ahmed Hachimi ${ }^{3}$
}

\author{
${ }^{1}$ Department of Hospitalary Pharmacist, Sidi Mohammed Ben Abdellah University in Fez, Morocco \\ ${ }^{2}$ Department of Emergency, Sidi Mohammed Ben Abdellah University in Fez, Morocco \\ ${ }^{3}$ Department of Anesthésie-Intensive Care, Sidi Mohammed Ben Abdellah University in Fez, Morocco
}

\author{
DOI: $10.36348 /$ sjmps.2020.v06i07.003 $\quad$ | Received: 28.06.2020 | Accepted: 06.07.2020 | Published: 21.07 .2020 \\ *Corresponding author: El Kartouti Abdeslam
}

\section{Abstract}

Objective: To evaluate the practice of antibiotic prophylaxis in trauma-orthopedic surgery at the Moulay Ismail military hospital in Meknes, in order to determine the deviations from the local protocol established and to propose corrective measures. Materials and Methods: This is a descriptive prospective study evaluating the practice of antibiotic prophylaxis in trauma and orthopedic surgery, which involved two hundred seventy-two patients operated on over a period of six months. This study took place in three phases. The collection of data on cards developed for this purpose; analysis of the results and review of the literature to judge the compliance of antibiotic prophylaxis decisions with local recommendations and protocols. Results: For the 272 patients operated on during the study period, of whom $72 \%$ were male, the compliance with the judgment criteria was respectively: $97.80 \%$ for the indication with antibiotic prophylaxis, $98.33 \%$ for the choice of molecule, $95 \%$ for the administered dose, $100 \%$ for the schedule of the first injection, $100 \%$ for the route of administration and only $3 \%$ for the duration of the antibiotic prophylaxis. Discussion and conclusion: The results of our study confirm the discrepancies reported by various international studies, these discrepancies are due to the deviation of antibiotic prophylaxis practices to local protocols and international standards. The antibiotic prophylaxis recommendations remain imperfectly applied even after the publication of guidelines. These results make essential: an update of the local protocols already established which must take into account on the one hand recent international recommendations and on the other hand local ecology, the day before the active dissemination of these protocols and their application by professionals and the need to train all prescribers using methods adapted to their professional constraints. Educate surgeons in the hospital department on the lack of interest in extending the prescription of antibiotic prophylaxis beyond 48 hours and in prescribing unjustified combinations.

Keywords: Assessment, antibiotic prophylaxis, trauma and orthopedic surgery.

Copyright @ 2020: This is an open-access article distributed under the terms of the Creative Commons Attribution license which permits unrestricted use, distribution, and reproduction in any medium for non-commercial use (NonCommercial, or CC-BY-NC) provided the original author and source are credited.

\section{INTRODUCTION}

Surgical site infection (ISO) is a nosocomial infection. This type of infection (ISO) remains a major challenge for public health. It is at the forefront in terms of morbidity for patients undergoing surgical procedures, leading on the one hand to an additional length of stay and an additional cost, and on the other hand to a serious sequelae which can go as far as the death of patients [1]. In France, infection of the operating site is the third most frequent nosocomial infection behind urinary tract infections according to the national survey on the prevalence of nosocomial infections [2].

Infection of the operating site is revealed within 30 days of surgery or within a year if an implant, prosthesis or prosthetic material is inserted [3, 4]. Infection of the operating site in trauma-orthopedics is a very serious complication, it can call into question the benefit of an intervention. Antibiotic prophylaxis is one of the essential tools for reducing the risk of infection in surgery. It does not eliminate the need to comply with basic hygiene measures and good surgical practices in which it must be integrated. Its purpose is to prevent possible infection of the operating site, reduce the emergence of multidrug-resistant bacteria and reduce unnecessary costs. Few countries have national statistics, and significant discrepancies can be observed depending on the establishment studied. This is the case of Morocco where few studies have been carried out to determine the importance and consequences of SSIs, particularly in trauma-orthopedic surgery. 
Antibiotic prophylaxis is a complementary measure in the fight against infection, which must be combined with other preventive measures: a preoperative skin preparation, a preoperative shower and hygienic measures to be taken in the operating room.

The objective of our work is to reassess the practice of antibiotic prophylaxis in trauma and orthopedic surgery, at the Moulay Ismail military hospital in Meknes, after the ATBP protocol was established, in order to verify compliance with it and determine deviations from this protocol and propose corrective measures.

\section{Materials ANd Methods Organization of the Study}

This is a prospective and descriptive study for the evaluation of antibiotic prophylaxis in trauma and orthopedic surgery at the My Ismail Military Hospital in Meknes, carried out by a work team made up of the pharmaceutical team and anesthetists - resuscitators, trauma-orthopedic surgeons and microbiologists, who was responsible for developing the methodology. The study took place in three phases:

1. The collection of data prospectively for each patient on files developed in consultation. This collection was carried out over a period of six months.

2. The analysis of the results made it possible to differentiate the criteria for inclusion and exclusion of the cards subject to antibiotic prophylaxis and to differentiate the cards with prescriptions for antibiotics with or without antibiotic prophylaxis.

3. Reading of literature reviews to judge the conformity of antibiotic prophylaxis decisions with regard to specific specialty recommendations based on the guidelines and their adaptations to local conditions.

\section{Choice of Study Periods}

Our prospective study was carried out over a period of six months, which allowed us to include 272 patients, a number allowing us to obtain interpretable results.

\section{Inclusion Criteria}

The prescriptions of all the patients who underwent a trauma-orthopedic surgery during the six months were noted thanks to the files drawn up and filled in by the anesthesiologists - resuscitators.

\section{Exclusion Criteria}

Patients on antibiotic therapy, Patients for whom the assessment was likely to be difficult, in particular patients at particular infectious risk (immunosuppressed or colonized by a nosocomial microbial flora); as well as class III and IV surgical procedures, the infection of which involves antibiotic therapy.

\section{Data Collection}

The information collected on the files concerned: - The patient: his name, first name, hospital service, allergy case, ASA score, hospitalization in the previous days, prior antibiotic; - The type of intervention, its date and the class of contamination; • The decision on antibiotic prophylaxis: -the 1st administration (specifying the antibiotic prescribed, the date, the time in relation to the incision, route of administration, dosage); -re-injections (specifying the antibiotic chosen, the number of doses, the duration, route of administration, and the dosage).

\section{Data Analysis}

The initial analysis of the data was conducted by the pharmaceutical team.

The prescriptions were considered to be compliant if they simultaneously met the following five judgment criteria;

1. Respect for the indication for antibiotic prophylaxis;

2. Appropriate choice of antibiotic and its route of administration;

3. Adequate timetable for the first administration;

4. Compliance of dosages;

5. Appropriate duration of treatment.

The results were compared to protocols based on the recommendations of the 2017 SFAR consensus conference on antibiotic prophylaxis in surgery. They were communicated orally to the various prescribers. They were then discussed by the entire team to determine the causes of the malfunctions can be highlighted.

The two fundamental axes on which the reflection for the updating of the protocols was based were:

1. The duration of antibiotic prophylaxis, reduced to 48 hours maximum for all types of surgery;

2. Imperative administration of antibiotics before the start of the surgical procedure.

The other goal is to try to revise the protocol already in place and to actively educate all stakeholders. The final version of the protocols was distributed to all medical teams and displayed in the operating rooms.

\section{RESUlTS \\ Study Population}

- During this study, 272 files were identified, including 180 files with prescription for antibiotic prophylaxis and 92 files without prescription for antibiotic prophylaxis. The gender distribution was predominantly by male with 195 records (72\%); the female sex with 
76 female files (28\%) and (sex ratio $\mathrm{M} / \mathrm{F}$ of 2.56).

- The distribution according to the ASA score showed that among the 272 patients; 224 patients have an ASA score $=1$, ie $(82.3 \%) ; 46$ Patients have an ASA score $=2(17 \%)$ and 2 patients have an ASA score $=3(0.79 \%)$.

- Altemeier's contamination class out of 272 interventions showed 255 interventions are in class 1 and 16 interventions is in class 2 .

\section{Types of interventions}

Trauma interventions (Figure-1)

Among the 272 patients studied, there were 237 cases of trauma interventions (87\%), of which 157 patients benefited from ATBP. They are dominated by ablation of osteosynthesis material $(30 \%)$ followed by per trochanteric fractures $(14 \%)$.

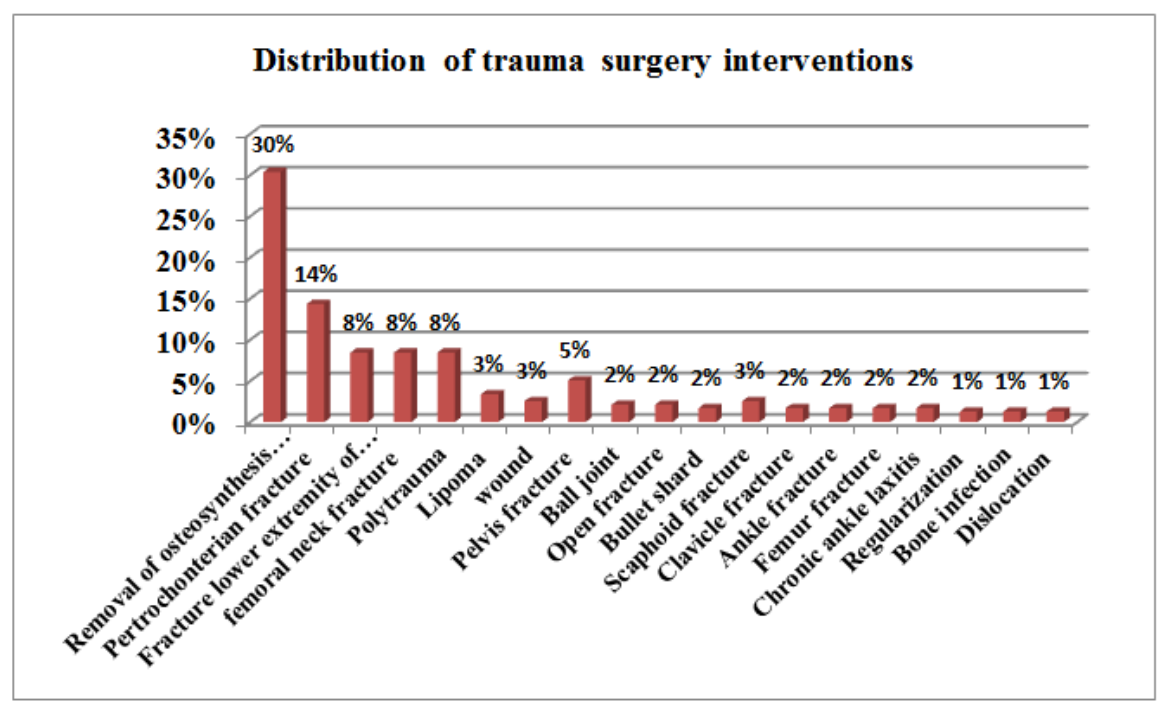

Fig-1: Percentage distribution of the different types of trauma interventions

\section{Orthopedic interventions (Figure-2)}

Among the 272 patients, there were 35 cases of orthopedics (13\%); grouping total hip prostheses (PTH), total knee prostheses (PTG), osteotomy of varisation or valgisation (OTV) and arthroscopy of the knee essentially and distributed as follows: - PTH: 13 cases - PTG: 6 cases - OTV: 4 cases • ARTHROSCOPY: 12 cases Among these 35 cases only the 12 cases of arthroscopy did not receive antibiotic prophylaxis according to the recommendations.

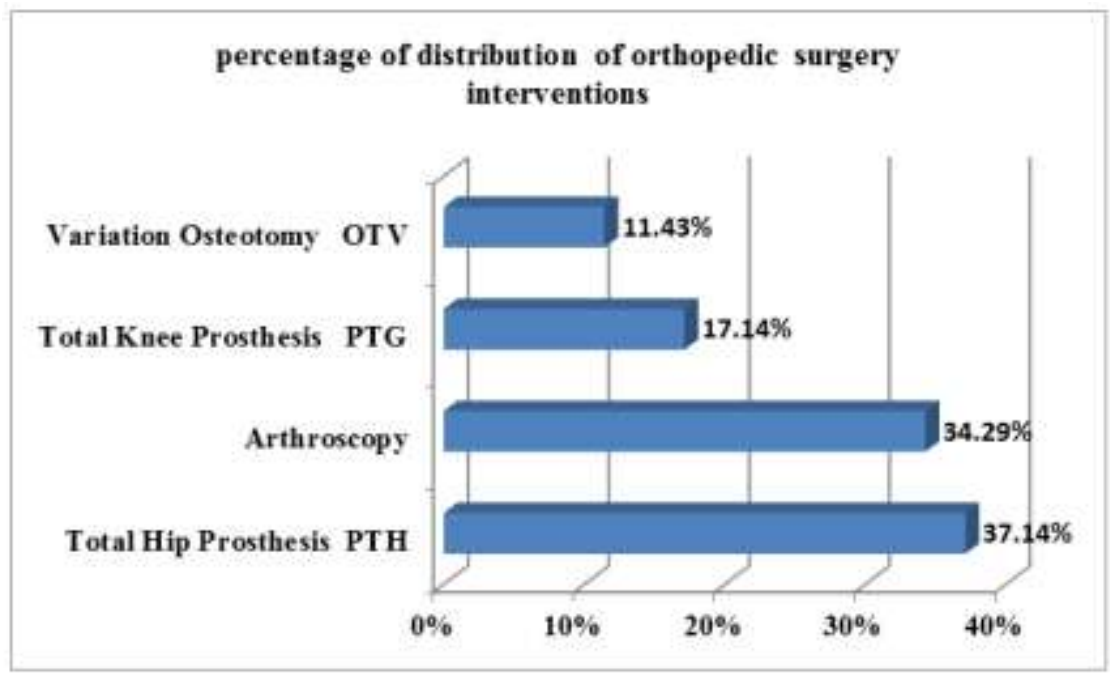

Fig-2: Percentage distribution of the different types of orthopedic intervention

\section{Prescribed Antibiotics}

In the 180 patients who received antibiotic prophylaxis; the antibiotics prescribed were dominated by Cefalotine $1 \mathrm{~g}$ in $86 \%$ of the cases; followed by protected amoxicillin in $4 \%$ of cases (Figure-3). 


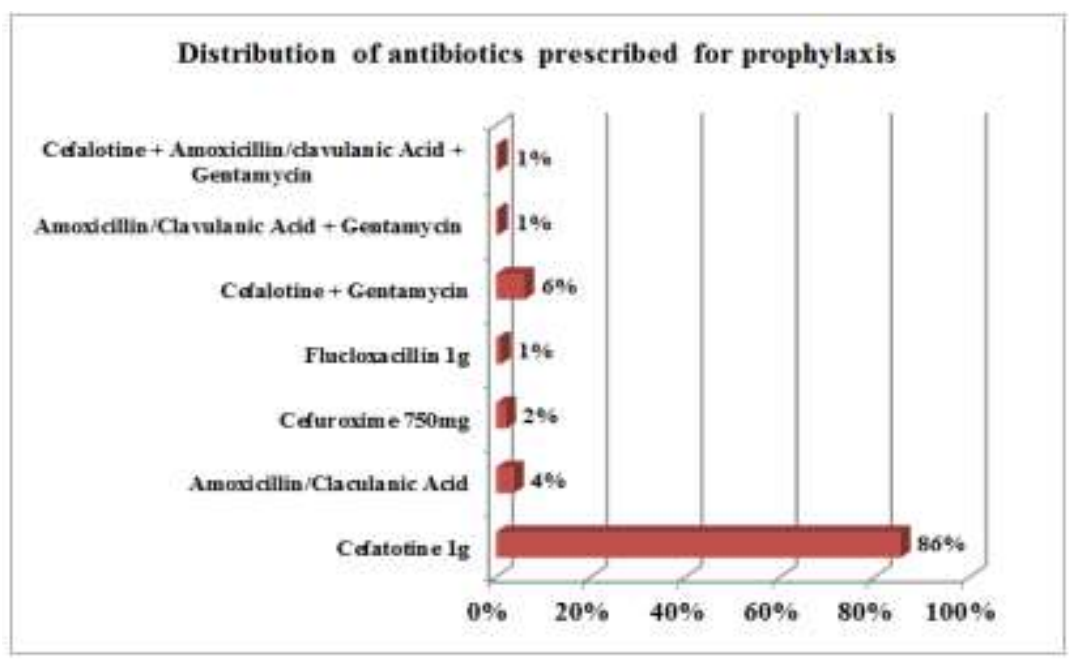

Fig-3: Percentage distribution of prescribed antibiotics

\section{Post - operative antibioprophylaxis}

The 180 patients who benefited from antibiotic prophylaxis, all received a second dose of antibiotic in the immediate post-operative period, the prescribed molecule was amoxicillin protected at a dose of $2 \mathrm{~g}$ administered by direct intravenous route.

\section{Antibiotherapy after 48 hours}

\section{Prescription of antibiotic therapy after $\mathbf{4 8}$ hours}

175 patients or $97 \%$ received antibiotic therapy after 48 hours and only 5 patients received nothing.

\section{The Dosage}

The prescribed drug was protected amoxicillin $\lg 3$ times daily for all patients.

\section{The Route of Administration}

Antibiotic therapy after 48 hours was administered orally in 164 patients or $96 \%$ and intravenously in 7 patients.

\section{Conformity of the antibioprophylaxis adjuging criteria}

In the 180 cases who received antibiotic prophylaxis:

- The compliance rate of the indication for antibiotic prophylaxis was in our study of $97.80 \%$.

- The compliance rate for the choice of antibiotic was $98.33 \%$.

- The compliance of the dosages of the antibiotics administered was $95 \%$ of the cases (all the dosages of the unsuitable antibiotics were considered as non-compliant).

- The compliance with the adequate schedule for the first administration was $100 \%$ compliant, the route of administration was also $100 \%$ compliant.

- The conformity of the duration of antibiotic prophylaxis which must not exceed 48 hours according to the recommendations of the SFAR; was only $3 \%$ because $175 / 180$ patients received a prescription for antibiotics after 48 hours.

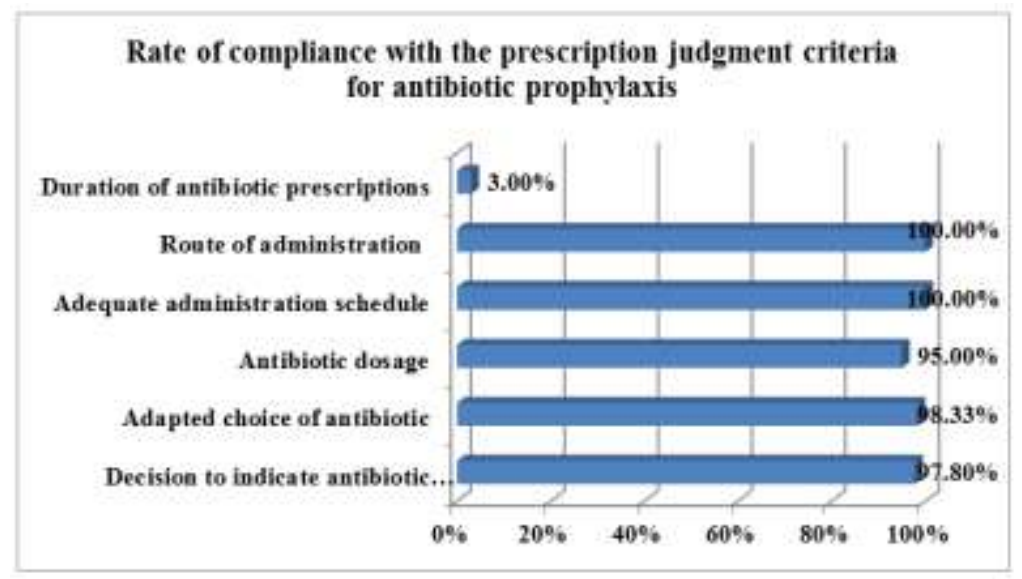

Fig-4: Rate of compliance of judgment criteria for antibiotic prophylaxis prescriptions

\section{DisCUSSION}

Antibiotic prophylaxis is one of the essential tools for reducing the risk of infection in surgery. It is possible to define this antibiotic prophylaxis according to the type of procedure planned, the infectious risk which relates to it and to adapt the prescription 
according to the history, any anti-infectious treatments already in progress, or finally in case of known carriage of a microorganism potentially dangerous for the patient or the community. As part of the improvement of prescription practices for antibiotic prophylaxis in surgery at the Moulay Ismail military hospital in Meknes, it was considered important to conduct this prospective study to reassess the practice of antibiotic prophylaxis in traumatic surgery. Orthopedic, to assess compliance with protocols, determine deviations from international standards and propose corrective measures.

\section{The conformity of the practice of antibiotic prophylaxis}

This study made it possible to make the observation concerning the application of the protocols established. The results of this study showed the persistence of discrepancies in antibiotic prophylaxis practices despite the improvement in the scores of certain endpoints. The patients included in our study were male in $72 \%$ and $28 \%$ female, the morbidity rate was low (only $1 \%$ had an ASA score $=3$ and no patient had an ASA score $=4$ ), most patients were admitted for trauma surgery in $87 \%$ of the cases.

\section{The conformity of the indication for antibiotic prophylaxis}

In our study, with that of the 2016 evaluation study carried out at our establishment, and other international studies has shown: a clear improvement in this criterion in our study $(97.80 \%)$ compared to the 2016 evaluation study $(92.8 \%)$ [5]. This rate of compliance of the indication in our study is correct compared to the data in the literature, which varies from $58.67 \%$ to $99.3 \%[6,7]$.

\section{The conformity of the choice of antibiotic}

Antibiotic prophylaxis must be addressed to a defined bacterial target, recognized as the most frequently involved in the infection of the operating site for the procedure concerned. The ABP protocol must include a molecule that includes this bacterial target in its spectrum [8]. He should not try to take into account all the bacteria possibly encountered.

Methodologically acceptable work must have validated its activity, its local distribution and its tolerance in this indication. It is essential to select molecules with a narrow spectrum of activity and which have obtained a MA in this indication. The rules to follow for choosing the antibiotic are:

- Effective on potentially contaminating germs;

- The diffusion at effective concentration in the tissue site concerned;

- Full dose administration;

- Administration before the risky act;

- Administration before induction;

- The order when the exposure to risk ceases;
- Have as few side effects as possible;

- Have the lowest possible cost;

- Not to be used in curative antibiotic therapy (as far as possible).

According to the updated recommendations of the SFAR, we note the abolition of the prescription of gentamicin previously recommended in combination with vancomycin in orthopedic surgery for subjects allergic to betalactamines [8]. At our establishment, the choice of Cefalotine $1 \mathrm{~g}$ and Cefuroxime $750 \mathrm{mg}$ has been validated by COMEDIMS. The results regarding the choice of antibiotics prescribed during our study showed that Cefalotine was the most prescribed molecule in $86 \%$ of the cases, followed by the protected amoxicillin in $4 \%$ of the cases. The compliance rate of the choice of antibiotic was overall (98\%), while the data in the literature report compliance rates varying from $23.80 \%$ to $82 \%$ [5, 6, 9].

\section{Conformity of the dosage of the antibiotic}

The initial dose or the loading dose of the antibiotic is usually double the usual dose. This recommendation applies up to a weight of $100 \mathrm{~kg}$ (the pharmacokinetic data make it possible to be sure of obtaining sufficient tissue concentrations of antibiotic). In the obese patient (patient over $100 \mathrm{~kg}$ and body mass index $>35 \mathrm{~kg} / \mathrm{m} 2$ ), even outside bariatric surgery, the doses of betalactamines must be double those recommended for non-obese patients.

The compliance of the dosage was (95\%), normally the initial dose should be double the usual dose according to the recommendations of the SFAR [10], this ensures a sufficient local concentration during the entire procedure with a single administration, even if diffusion is diminished by local conditions [8], an insufficient dosage is a recognized risk factor for postoperative infection, whether it is a dose.

By comparing the compliance of the dosage in our study with that of the 2016 evaluation study carried out at our establishment and other international studies: We note an improvement in the compliance rate of the dosage in our study (95\%) compared to the 2016 evaluation study $(23.08 \%)$ [5], but this rate remains correct given the contribution to international studies.

\section{The conformity of the schedule and the route of administration of antibiotic prophylaxis}

The time of prescription has been the subject of a very important debate in recent years [8]. To be effective in prophylaxis, an antibiotic must be present on the potentially contaminated site before contamination. The ABP must therefore always precede the incision within 30 to 60 minutes [8]. This point is fundamental. When using Vancomycin, the infusion should be started early enough to be completed 30 minutes before the procedure. The ABP must be 
administered 5 to $10 \mathrm{~min}$ before the anesthetic induction so that, in the event of an allergic reaction, we can make the share of what belongs to each. The definition of ABP (molecule and dose) in anesthesia consultation is fundamental. This anticipation will allow the IADE to start the infusion of the antibiotic prescribed as soon as the VVP is applied, and therefore to better respect the time necessary for the diffusion of the antibiotic before the incision. This is a very important criterion (the injection of the antibiotic must precede the incision within 30 to $60 \mathrm{~min}$ ), failure to comply with this period may contribute to an increased risk of SSI [11, 12], according to a study by Classen, the rate of infection of the operating site can be multiplied by four if this injection took place after the surgical incision [13]. The comparison of the conformity of the schedule for the first injection in our study with that of the 2016 evaluation study carried out at our establishment made it possible to conclude that there was total compliance with this criterion in our study $(100 \%)$, as in the 2016 evaluation study (100\%) [5]. The compliance rate for the schedule of the first injection in our study is optimal compared to other international studies, the rate varying between $56.3 \%$ to $96 \%$ [6, 9]. For the route of administration, this criterion has been adapted in $100 \%$ of cases.

\section{The conformity of the duration of treatment}

The efficacy of antibiotic prophylaxis is no longer to be demonstrated; a study reports that the rate of infection of the locked nailing for closed fracture (leg and thigh combined) went from $4.4 \%$ without antibiotic prophylaxis to $2.2 \%$ with antibiotic prophylaxis. However, in some studies the antibiotic prescribed was Cefazolin pre and postoperative and the duration of prescription was 5 days. Whereas the recommendations consist in stopping the $\mathrm{ABP}$ as soon as the risk of contamination ceases. After the initial dose, reinjections are given during the operating period, every two half-lives of the antibiotic, at a dose that is either similar or half the initial dose. In practice, the ABP must be brief, generally limited to the operating period, sometimes 24 hours and exceptionally 48 hours and never beyond. Even the presence of drainage from the operating room does not allow us to transgress these recommendations. There is no reason to prescribe reinjections when removing drains, catheters or catheters.

Regarding the results of our study, the duration of antibiotic prophylaxis was inadequate in $97 \%$ of the cases. The antibiotic coverage of the surgical procedure was generally correct, but the duration of treatment was extended beyond $48 \mathrm{~h}$ in 175 cases according to the results of our study, by comparing the conformity of the total duration of the antibiotic prophylaxis of our study. , with that of the 2016 reassessment carried out at our establishment and other international studies, we note a clear drop in the compliance rate of the total duration of ATBP in our study (3\%) compared to the 2016 evaluation study (53.80\%) [5], while a French multicentre study reports a compliance rate of $77.4 \%$ [6]. Whereas, another French study confirms this trend of postoperative prescription in a hospital center by reporting an unjustified and or too long prescription rate in $87 \%$ of patients [14]. These results thus show that the imperfections and discrepancies with respect to the recommendations are in the supposed sense of an individual benefit of a patient, compared to the collective benefit desired by respecting the rules of antibiotic prophylaxis, by an excess of prescription, by unjustified associations, by the extension of the duration and sometimes by the use of molecules with a wider spectrum. Whereas the aforementioned prescription for antibiotic prophylaxis beyond 48 hours promotes the appearance of resistance according to the data in the literature and increases the costs [15].

Table-1: Comparison of the conformity of the criteria for judgment of antibiotic prophylaxis

\begin{tabular}{|l|l|l|l|l|l|l|l|}
\hline Studies & $\begin{array}{l}\text { Number } \\
\text { of } \\
\text { patients }\end{array}$ & Duration & $\begin{array}{l}\text { Conformity } \\
\text { of } \\
\text { indication }\end{array}$ & $\begin{array}{l}\text { Conformity } \\
\text { of antibiotic } \\
\text { choice }\end{array}$ & $\begin{array}{l}\text { Conformity } \\
\text { of dosage }\end{array}$ & $\begin{array}{l}\text { Conformity } \\
\text { of timetable }\end{array}$ & $\begin{array}{l}\text { Conformity } \\
\text { of duration }\end{array}$ \\
\hline $\begin{array}{l}\text { French Aquitaine multicentre } \\
\text { evaluation study in 2007 [6] }\end{array}$ & 9651 & $\begin{array}{l}12 \\
\text { months }\end{array}$ & $99.3 \%$ & $82 \%$ & $85.40 \%$ & $56.30 \%$ & $77.40 \%$ \\
\hline $\begin{array}{l}\text { Tunisian study CHU of Tunis in } \\
2015 \text { [9] }\end{array}$ & 150 & 1 month & $74 \%$ & $82 \%$ & $96 \%$ & $96 \%$ & $28 \%$ \\
\hline $\begin{array}{l}\text { Iranian study Nemazi Hospital in } \\
\text { Shiraz 2010 [13] }\end{array}$ & 166 & 2 weeks & $68.40 \%$ & $7.50 \%$ & & $76.50 \%$ & $41.10 \%$ \\
\hline $\begin{array}{l}\text { Ethiopian Study North Ayder } \\
\text { Hospital 2016 [7] }\end{array}$ & 196 & 6 weeks & $58.67 \%$ & $10.50 \%$ & $89.60 \%$ & & $36.50 \%$ \\
\hline $\begin{array}{l}\text { Antibiotic prophylaxis } \\
\text { assessment study military } \\
\text { hospital moulay ismail 2016 [5] }\end{array}$ & $\mathbf{3 0}$ & $\begin{array}{l}\mathbf{2} \\
\text { months }\end{array}$ & $\mathbf{9 2 . 8 6 \%}$ & $\mathbf{2 3 . 8 0 \%}$ & $\mathbf{2 3 . 0 8 \%}$ & $\mathbf{1 0 0 \%}$ & $\mathbf{5 3 . 8 0 \%}$ \\
\hline $\begin{array}{l}\text { Antibiotic prophylaxis } \\
\text { reassessment study military } \\
\text { hospital moulay ismail 2018 }\end{array}$ & $\mathbf{2 7 2}$ & $\begin{array}{l}\mathbf{6} \\
\text { months }\end{array}$ & $\mathbf{9 7 . 8 0 \%}$ & $\mathbf{9 8 \%}$ & $\mathbf{9 5 \%}$ & $\mathbf{1 0 0 \%}$ & $\mathbf{3 \%}$ \\
\hline
\end{tabular}

The results obtained during our study show discrepancies with the antibiotic prophylaxis protocol by not respecting the local protocol adapted and displayed for each type of intervention. In order to correct the dysfunctions and make improvements to the prescription of surgical antibiotic prophylaxis, it was 
necessary; update the written protocols for all interventions in trauma and orthopedic surgery. The protocols must take into account on the one hand the specific local ecology of the trauma-orthopedic service. The various authors also advise not to be satisfied with a simple passive dissemination of prescription recommendations, and confirm the low effectiveness of isolated educational methods in the context of the correct use of antibiotics to obtain an improvement in practices [17, 18]. Improvement in quality is also conditioned by the involvement of all health professionals before surgery, at the time of the intervention and in the care service [19]. On the other hand, the association of an educational policy (organization of training meetings for the various actors in antibiotic prophylaxis, display of the protocols developed ...) with the dissemination of local consensus would certainly be desirable. Other studies have shown that the computer tool plays an important role in improving the practice of antibiotic prophylaxis and the adequacy of local protocols; it is a tool for decision support, rationalization and of professional practices [20].

\section{Conclusion}

Surgical infections account for a quarter of nosocomial infections. They are an important cause of morbidity, and increase postoperative mortality. The incidence and cost of complications from surgical site infections are difficult to assess. Antibiotic prophylaxis remains one of the essential means for reducing this risk of infection. The combination of other means such as basic hygiene measures and good surgical practices is essential. Our prospective and descriptive study included 272 patients, the overall analysis of the results showed that the overall decision compliance rate for antibiotic prophylaxis was $97.22 \%$, these results are close to those of the literature. Regarding the analysis of the judgment criteria, the results showed the persistence of discrepancies with respect to the antibiotic prophylaxis rules, the criterion of prescription duration was the least suitable criterion. Two points must be the subject of particular attention from resuscitation anesthesiologists as well as surgeons: the frequency of prescriptions in excess beyond 48 hours and the use of unjustified combinations. Hence the interest in revising local protocols and monitoring their compliance.

\section{REFERENCES}

1. VASSELLE Alain; Rapport de l'OPEPS $n^{\circ} 421$ (2005-2006).

2. Thiolet, J. M., Vaux, S., Lamy, M., Gautier, A., Barret, A. S., Léon, L., \& Coignard, B. (2012). Enquête nationale de prévalence des infections nosocomiales et des traitements anti-infectieux en établissements de santé. France, mai-juin.

3. Beaucaire, G. (1997). Infections nosocomiales:
Épidémiologie, critères du diagnostic, prévention, principes de traitement. La Revue du praticien (Paris), 47(2), 201-209.

4. Berche, P., Gallard, J. L., \& Simonnet, M. (1991). Les infections nosocomiales d'origine bactérienne et leur prévention. Bactériologie des infections humaines de la biologie à la clinique. Paris: Flammarion, 64-71.

5. Salma, M. E. (2017). Evaluation de la pratique de l'antibioprophylaxie en chirurgie Expérience de l'hôpital militaire Moulay Ismail De Meknes (A PROPOS DE 216 CAS).

6. Maury, B., Dupon, C. D., Dupon, M., Labat, A., \& Kosellek, D. (2007). Evaluation of antibiotic prophylaxis for hip and knee replacement: a multicentered study in Aquitaine public and private hospitals (SouthWestern France). Medecine et Maladies Infectieuses, 37(3), 166-171.

7. Mohamoud, S. A., Yesuf, T. A., \& Sisay, E. A. (2016). Utilization assessment of surgical antibiotic prophylaxis at Ayder Referral Hospital, Northern Ethiopia. J Appl Pharm, 8(2).

8. Gilles, L., Favier, B., \& Latour, J. (2002). Survey of antimicrobial prophylaxis practices in surgery. Journal de Pharmacie Clinique, 21: 91-98.

9. Harbi, H., Merzougui, L., Barhoumi, M. H., Rebai, H., Abdelkefi, S., El Kamel, R., \& Barhoumi, T. (2018). Evaluation des pratiques d'antibioprophylaxie chirurgicale dans un Hopital Universitaire du Centre Tunisien. The Pan African Medical Journal, 30.

10. Mangram, A. J., Horan T. C., \& Pearson, M. L. (1999). Guideline for prevention of surgical site infection, 1999. Hospital Infection Control Practices Advisory Committee. Infect Control Hosp Epidemiol. 20:250-278.

11. DiPiro, J. T., Cheung, R. P., Bowden Jr, T. A., \& Mansberger, J. A. (1986). Single dose systemic antibiotic prophylaxis of surgical wound infections. The American journal of surgery, 152(5), 552-559.

12. Classen, D. C., Evans, R. S., Pestotnik, S. L., Horn, S. D., Menlove, R. L., \& Burke, J. P. (1992). The timing of prophylactic administration of antibiotics and the risk of surgical-wound infection. New England Journal of Medicine, 326(5), 281-286.

13. Vessal, G., Namazi, S., Davarpanah, M. A., \& Foroughinia, F. (2011). Evaluation of prophylactic antibiotic administration at the surgical ward of a major referral hospital, Islamic Republic of Iran. EMHJ-Eastern Mediterranean Health Journal, 17 (8), 663-668, 2011.

14. De Bortoli, C., Meyer, M., Arnaud, V., Novotny, R., Manoli, P., Comparot, S., ... \& Bastia, B. (2014). État des lieux et pistes d'amélioration des pratiques de l'antibioprophylaxie chirurgicale à l'échelle d'un centre hospitalier. Le Pharmacien 
Hospitalier et Clinicien, 49(4), 327-328.

15. Agence Nationale d'accréditation et d'évaluation en santé. (2000). Octobre 2000. Evaluation des pratiques professionnelles dans les établissements de santé. Evaluation de l'antibioprophylaxie en chirurgie propre: application à la prothèse totale de hanche octobre.

16. Société française d'anesthésie et de réanimation. (2018). Recommandations pour la pratique de l'antibioprophylaxie en chirurgie. Conférence.

17. d'Escrivan, T., Lemaire, J. S., Ivanov, E., Boulo, M., Soubrier, S., Mille, F. X., ... \& Guery, B. (2005, January). Antibioprophylaxie chirurgicale: adéquation aux recommandations et impact d'une action d'information ciblée. In Annales francaises d'anesthesie et de reanimation (Vol. 24, No. 1, pp. 19-23). Elsevier Masson.

18. Maury, B., Dupon, C. D., Dupon, M., Labat, A., \&
Kosellek, D. (2007). Évaluation de l'antibioprophylaxie pour implantation de prothèse de hanche et de genou: une étude multicentrique dans les établissements de santé d'Aquitaine (France). Médecine et maladies infectieuses, 37(3), 166-171.

19. Carlès, M., Gindre, S., Aknouch, N., Goubaux, B., Mousnier, A., \& Raucoules-Aimé, M. (2006). Improvement of surgical antibiotic prophylaxis: a prospective evaluation of personalized antibiotic kits. Journal of Hospital Infection, 62(3), 372-375.

20. Fayolle-Pivot, L., Wey, P. F., Petitjeans, F., Puidupin, M., Allaouchiche, B., \& Escarment, J. (2013, April). Apport de l'outil informatique dans l'application de protocoles: exemple de l'antibioprophylaxie chirurgicale. In Annales francaises d'anesthesie et de reanimation (Vol. 32, No. 4, pp. 241-245). Elsevier Masson. 